\title{
On the equivalence of two fundamental theta identities
}

\author{
Tom H. Koornwinder \\ Dedicated to the memory of Frank W. J. Olver
}

\begin{abstract}
Two fundamental theta identities, a three-term identity due to Weierstrass and a fiveterm identity due to Jacobi, both with products of four theta functions as terms, are shown to be equivalent. One half of the equivalence was already proved by R. J. Chapman in 1996. The history and usage of the two identities, and some generalizations are also discussed.
\end{abstract}

\section{Introduction}

Theta functions occur in many parts of mathematics and its applications [17]. While they had roots in the work of Jakob Bernoulli and Euler, they were introduced in full generality, depending on two arguments, by Jacobi. They became very important in nineteenth century complex analysis [6], 25. Ch. 11] because elliptic functions could be expressed in terms of them. Theta functions in several variables, later called Riemann theta functions [34, §21.2], played a similar role for abelian functions. Riemann's geometric approach [35] and Weierstrass' analytic approach [51] were opposed to each other. Ramanujan, in Chapters 16-21 of his Notebooks, gave many new theta identities, with an emphasis on number theoretical and modular aspects; see Berndt [4] for an edited version with proofs. Algebraic geometry, number theory and combinatorics are some of the fields where theta functions have played an important role since long. New fields of application arose during the last decades of the twentieth century: nonlinear pde's like KdV [16], solvable models in statistical mechanics [3], Sklyanin algebra [45], 46], elliptic quantum groups [19] and elliptic hypergeometric series [21], [22, Ch. 11], 48].

In literature identities involving theta functions abound, see for instance Whittaker \& Watson [53. Ch. 21], Erdélyi et al. [18, §13.10] and Olver et al. [34, Ch. 20], but two identities (given in [53] and [34]) stand out because of their fundamental nature and because many of the other identities can be derived from them. Both have the form of a sum of products of four theta functions of different arguments being zero, with three terms in the first formula and five terms in the second formula.

First fundamental theta identity

$$
\begin{array}{r}
\theta_{1}\left(u+u_{1}\right) \theta_{1}\left(u-u_{1}\right) \theta_{1}\left(u_{2}+u_{3}\right) \theta_{1}\left(u_{2}-u_{3}\right)+\theta_{1}\left(u+u_{2}\right) \theta_{1}\left(u-u_{2}\right) \theta_{1}\left(u_{3}+u_{1}\right) \theta_{1}\left(u_{3}-u_{1}\right) \\
+\theta_{1}\left(u+u_{3}\right) \theta_{1}\left(u-u_{3}\right) \theta_{1}\left(u_{1}+u_{2}\right) \theta_{1}\left(u_{1}-u_{2}\right)=0
\end{array}
$$


(or equivalently with $\theta_{1}$ replaced by $\sigma$ ), see p.451, Example 5 and p.473, $\S 21.43$ in [53], or (23.10.4) and (23.6.9) in [34.

\section{Second fundamental theta identity}

$$
\begin{aligned}
2 \theta_{1}(w) \theta_{1}(x) \theta_{1}(y) \theta_{1}(z)=\theta_{1}\left(w^{\prime}\right) & \theta_{1}\left(x^{\prime}\right) \theta_{1}\left(y^{\prime}\right) \theta_{1}\left(z^{\prime}\right)+\theta_{2}\left(w^{\prime}\right) \theta_{2}\left(x^{\prime}\right) \theta_{2}\left(y^{\prime}\right) \theta_{2}\left(z^{\prime}\right) \\
& -\theta_{3}\left(w^{\prime}\right) \theta_{3}\left(x^{\prime}\right) \theta_{3}\left(y^{\prime}\right) \theta_{3}\left(z^{\prime}\right)+\theta_{4}\left(w^{\prime}\right) \theta_{4}\left(x^{\prime}\right) \theta_{4}\left(y^{\prime}\right) \theta_{4}\left(z^{\prime}\right),
\end{aligned}
$$

where

$$
\begin{aligned}
2 w^{\prime} & =-w+x+y+z, & & 2 x^{\prime}=w-x+y+z, \\
2 y^{\prime} & =w+x-y+z, & & 2 z^{\prime}=w+x+y-z
\end{aligned}
$$

and similar equivalent identities starting with $\theta_{2}, \theta_{3}$ or $\theta_{4}$ on the left-hand side, see $\S 21.22$ in [53], or (21.6.6) and (21.2.9) in [34]

Identity (1.2) (the oldest one) was first given by Jacobi [27, p.507, formula (A)]; this paper is based on notes made by Borchardt of a course of Jacobi which were later annotated by Jacobi. It first entered in Jacobi's lectures of 1835-1836 and he was so excited by the result that he completely changed his approach to elliptic functions, using (1.2) as a starting point [6, p.220].

Identity (1.1) was first obtained by Weierstrass [50, (1.)]. For the proof he refers to Schwarz [43. Art. 38, formula (1.)] (these are edited notes of lectures by Weierstrass). Weierstrass [50] mentions that he first gave this formula in his lectures in 1862. He emphasizes that (1.1) is essentially different from Jacobi's formulas (1.2) and variants.

Some papers in the last decades have attributed these formulas to Riemann, although without reference. Frenkel \& Turaev [21, pp. 171-172] call formula (1.1) Riemann's theta identity. Some later authors [40, (3.4)], [38, (5.3)], [48, (6)] also use this terminology or speak about Riemann's addition formula. As for (1.2), Mumford [32, [33, p.16] calls it Riemann's theta relation. However, I have not been able to find formula (1.1) or (1.2) in 35 or elsewhere in Riemann's publications [36].

Formula (1.2) has a generalization [33, Ch. 2, §6], [34, §21.6(i)] to theta functions in several variables, which is called a generalized Riemann theta identity by Mumford. Weierstrass [50], 52 gave a generalization of both (1.1) and (1.2), respectively, to the several variables case. It is not immediately clear how the results in [33] and [52] are related.

The main purpose of this paper is to show in Section 5 that (1.1) and (1.2) easily follow from each other, and therefore can be considered to be equivalent identities. We will work in the notation [22, (11.2.1)] for theta functions which is now common in work on elliptic hypergeometric series. Its big advantage is that we have only one theta function instead of four different ones, by which lists of formulas can be greatly shrinked. Another feature of this notation is that we work multiplicatively instead of additively. Instead of double (quasi-)periodicity we have quasiinvariance under multiplication of the independent variable by $q$. This notation is introduced in Section 2. Some variants and applications of the two fundamental formulas are given in Section 3. For completeness the elegant proofs by complex analysis of the two fundamental formulas 
are recalled in Section 4 and some other proofs are mentioned. An Appendix compares some four-term theta identities (mostly extending (1.1)).

During the revision of an earlier version of this paper my attention was called to work by Wenchang Chu. Apparently unaware of the earlier occurrence of (1.1) in the literature he posed it as a problem in the Monthly to prove this formula (as a solution to a functional equation). Several authors gave solutions [9]. One of them, R. J. Chapman, derived (1.1) from (1.2), essentially the same proof as my proof in Section 5. Thus the present paper has become even more a survey paper than originally intended.

Acknowledgements I thank an anonymous referee for corrections of minor errors, for important additional material, and for posing a question which brought me to writing the Appendix. I also thank Bruce Berndt, Hjalmar Rosengren and Michael Schlosser for providing additional references, and Michael Somos for interesting comments.

\section{Preliminaries}

Let $q$ and $\tau(\bmod 2 \mathbb{Z})$ be related by $q=e^{i \pi \tau}$ and assume that $0<|q|<1$, or equivalently $\operatorname{Im} \tau>0$. We will define and notate the theta function of nome $q$ as in Gasper \& Rahman [22, $(11.2 .1)]$ :

$$
\begin{aligned}
\theta(w)=\theta(w ; q) & :=(w, q / w ; q)_{\infty}=\prod_{j=0}^{\infty}\left(1-q^{j} w\right)\left(1-q^{j+1} / w\right) \quad(w \neq 0), \\
\theta\left(w_{1}, \ldots, w_{k}\right) & :=\theta\left(w_{1}\right) \ldots \theta\left(w_{k}\right) .
\end{aligned}
$$

By Jacobi's triple product identity [22, (1.6.1)] we have

$$
\theta(w ; q)=\frac{1}{(q ; q)_{\infty}} \sum_{k=-\infty}^{\infty}(-1)^{k} q^{\frac{1}{2} k(k-1)} w^{k} .
$$

Clearly,

$$
\begin{aligned}
\theta\left(w^{-1} ; q\right) & =-w^{-1} \theta(w ; q), \\
\theta(q w ; q) & =-w^{-1} \theta(w ; q), \\
\theta\left(q^{k} w ; q\right) & =(-1)^{k} q^{-\frac{1}{2} k(k-1)} w^{-k} \theta(w ; q) \quad(k \in \mathbb{Z}) .
\end{aligned}
$$

The four Jacobi theta functions $\theta_{a}$ or $\vartheta_{a}(a=1,2,3,4)$, written as

$$
\theta_{a}(z)=\theta_{a}(z, q)=\theta_{a}(z \mid \tau)=\vartheta_{a}(\pi z, q)=\vartheta_{a}(\pi z \mid \tau)
$$


can all be expressed in terms of the theta function (2.1):

$$
\begin{aligned}
& \theta_{1}(z):=i q^{1 / 4}\left(q^{2} ; q^{2}\right)_{\infty} e^{-\pi i z} \theta\left(e^{2 \pi i z} ; q^{2}\right), \\
& \theta_{2}(z):=q^{1 / 4}\left(q^{2} ; q^{2}\right)_{\infty} e^{-\pi i z} \theta\left(-e^{2 \pi i z} ; q^{2}\right)=\theta_{1}\left(z+\frac{1}{2}\right), \\
& \theta_{3}(z):=\left(q^{2} ; q^{2}\right)_{\infty} \theta\left(-q e^{2 \pi i z} ; q^{2}\right)=\sum_{k=-\infty}^{\infty} q^{k^{2}} e^{2 \pi i k z} \\
& \theta_{4}(z):=\left(q^{2} ; q^{2}\right)_{\infty} \theta\left(q e^{2 \pi i z} ; q^{2}\right)=\theta_{3}\left(z+\frac{1}{2}\right) .
\end{aligned}
$$

Note that $\theta_{1}(z)$ is odd in $z$, while $\theta_{2}(z), \theta_{3}(z)$ and $\theta_{4}(z)$ are even in $z$.

The notation $\theta_{a}$ is used in [18, $\left.\S 13.10\right]$ and [34, Ch. 20], while the notation $\vartheta_{a}$ is used in [53, Ch. 21]. Mumford [33] writes $\vartheta(z, \tau)$ instead of $\theta_{3}(z \mid \tau)$.

The first fundamental identity (1.1) now takes the form

$$
y u \theta(x y, x / y, v u, v / u)+u v \theta(x u, x / u, y v, y / v)+v y \theta(x v, x / v, u y, u / y)=0,
$$

or variants by applying (2.4), see [22, (11.4.3)]. The terms in (2.7) are obtained from each other by cyclic permutation in $y, u, v$.

The second fundamental identity (1.2) can be rewritten in the notation (2.1) as

$$
\begin{aligned}
2 \theta\left(w^{2}, x^{2}, y^{2}, z^{2} ;\right. & \left.q^{2}\right)=\theta\left(w^{\prime \prime}, x^{\prime \prime}, y^{\prime \prime}, z^{\prime \prime} ; q^{2}\right)+\theta\left(-w^{\prime \prime},-x^{\prime \prime},-y^{\prime \prime},-z^{\prime \prime} ; q^{2}\right) \\
& +q^{-1} x y z w\left(\theta\left(q w^{\prime \prime}, q x^{\prime \prime}, q y^{\prime \prime}, q z^{\prime \prime} ; q^{2}\right)-\theta\left(-q w^{\prime \prime},-q x^{\prime \prime},-q y^{\prime \prime},-q z^{\prime \prime} ; q^{2}\right)\right),
\end{aligned}
$$

where

$$
w^{\prime \prime}=w^{-1} x y z, \quad x^{\prime \prime}=w x^{-1} y z, \quad y^{\prime \prime}=w x y^{-1} z, \quad z^{\prime \prime}=w x y z^{-1} .
$$

\section{Variants and applications of the two fundamental formulas}

As already observed in Section 1, Weierstrass wrote (1.1) as

$$
\begin{aligned}
\sigma\left(u+u_{1}\right) \sigma\left(u-u_{1}\right) \sigma\left(u_{2}+u_{3}\right) \sigma\left(u_{2}-u_{3}\right) & +\sigma\left(u+u_{2}\right) \sigma\left(u-u_{2}\right) \sigma\left(u_{3}+u_{1}\right) \sigma\left(u_{3}-u_{1}\right) \\
+ & \sigma\left(u+u_{3}\right) \sigma\left(u-u_{3}\right) \sigma\left(u_{1}+u_{2}\right) \sigma\left(u_{1}-u_{2}\right)=0 .
\end{aligned}
$$

The two formulas (1.1) and (3.1) are equivalent because by [53, p.473, §21.43], for periods 1 and $\tau$, we have $\sigma(z)=C e^{\eta_{1} z^{2} / 2} \theta_{1}\left(\frac{1}{2} z \mid \tau\right)$ with $C$ and $\eta_{1}$ only depending on $\tau$. For $v=1$, $u=-1$ formula (2.7) yields (using (2.4) ):

$$
\frac{y \theta(x y, x / y)}{\theta(x)^{2} \theta(y)^{2}}=f(y)-f(x), \quad \text { where } f(x):=\frac{\theta(-x)^{2}}{\theta(-1)^{2} \theta(x)^{2}} .
$$

Conversely, (3.2) implies (2.7), for any choice of the function $f$. Indeed, after two substitutions

from (3.2), the first term of (2.7) becomes $(\theta(x) \theta(y) \theta(u) \theta(v))^{2}(f(y)-f(x))(f(u)-f(v))$, which adds up to 0 under cyclic permutation of $y, u, v$. 
Weierstrass, according to Schwarz [43, Art. 38, formula (1.)], derived (3.1) from the formula [43, Art. 11, formula (1.)]:

$$
\frac{\sigma(u+v) \sigma(u-v)}{\sigma^{2}(u) \sigma^{2}(v)}=\wp(v)-\wp(u),
$$

where $\wp(z)$ is Weierstrass' elliptic function. By the expression [18, 13.20(4)] of $\wp(z)$ in terms of theta functions, (3.3) is equivalent to (3.2) just as (3.1) is equivalent to (2.7). Whittaker \& Watson give these results in [53, p.451, Examples 1 and 5]. Formula (3.3) is also given in [18, $(10.13 .17)]$ and [34, (23.10.3)].

All addition formulas for theta functions in [53, pp. 487-488, Examples 1, 2, 3] are instances of (3.2) or slight variants of it which can be obtained by specialization of (2.7). Some of these formulas are used in the proof that certain actions of the generators of the Sklyanin algebra on the space of meromorphic functions determine a representation of the Sklyanin algebra 46, Theorem 2].

Weierstrass [50] observed at the end of his paper that (3.1), as a functional equation for the sigma function, has a general solution given by a power series and still depending on four arbitrary constants. This was finally proved in full rigor by Hurwitz [26]. However, [53, pp. 452, 461] gives earlier references for this result to books by Halphen and by Hermite.

A stronger characterization of the sigma function (up to trivial transformations) and its degenerate cases was given by Bonk [5] as continuous solutions $\tau: \mathbb{R}^{n} \rightarrow \mathbb{C}$ of the functional equation

$$
\tau(u+v) \tau(u-v)=f_{1}(u) g_{1}(v)+f_{2}(u) g_{2}(v),
$$

where $f_{1}, f_{2}, g_{1}, g_{2}: \mathbb{R}^{n} \rightarrow \mathbb{C}$ are arbitrary. Note that (3.3) is of the above form.

Elliptic, and in particular theta functions, entered in work on solvable models in statistical mechanics started by Baxter [3] and followed up in papers like [1], 13], [14. While building on these publications, Frenkel \& Turaev [21] in their work on the elliptic $6 j$-symbol introduced elliptic hypergeometric series. Among others, they obtained the summation formula of the terminating well-poised theta hypergeometric series ${ }_{10} V_{9}\left(a ; b, c, d, e, q^{-n} ; q, p\right)$. Formula (2.7) occurs as the first non-trivial case $n=1$ and it also plays a role in the further proof by induction of this summation formula [22, §11.4]. Closely related to these developments is the introduction of elliptic quantum groups by Felder [19]. Again theta functions play here an important role [20], 29]. In [29, Remarks 2.4, 4.3] formula (2.7) is used in connection with the representation theory of the elliptic $U(2)$ quantum group.

If we pass in (2.7) to homogeneous coordinates $\left(a_{1}, a_{2}, a_{3}, b_{1}, b_{2}, b_{3}\right)$ satisfying $a_{1} a_{2} a_{3}=b_{1} b_{2} b_{3}$ and expressed in terms of $x, y, u, v$ by

$$
a_{1}=b_{3} x u, a_{2}=b_{3} x y, a_{3}=b_{3} x v, b_{1}=b_{3} x^{2}, b_{2}=b_{3} x y u v,
$$

then, after repeated application of (2.4), we obtain another symmetric version of (2.7):

$$
\frac{\theta\left(a_{1} / b_{1}, a_{1} / b_{2}, a_{1} / b_{3}\right)}{\theta\left(a_{1} / a_{2}, a_{1} / a_{3}\right)}+\frac{\theta\left(a_{2} / b_{1}, a_{2} / b_{2}, a_{2} / b_{3}\right)}{\theta\left(a_{2} / a_{3}, a_{2} / a_{1}\right)}+\frac{\theta\left(a_{3} / b_{1}, a_{3} / b_{2}, a_{3} / b_{3}\right)}{\theta\left(a_{3} / a_{1}, a_{3} / a_{2}\right)}=0 \quad\left(a_{1} a_{2} a_{3}=b_{1} b_{2} b_{3}\right) .
$$


Formula (3.4) has an $n$-term generalization which is associated with root system $A_{n-1}$ :

$$
\sum_{k=1}^{n} \frac{\prod_{j=1}^{n} \theta\left(a_{k} / b_{j}\right)}{\prod_{j \neq k} \theta\left(a_{k} / a_{j}\right)}=0 \quad\left(a_{1} \ldots a_{n}=b_{1} \ldots b_{n}\right),
$$

see [47, (5)], [15, Lemma A.2], [37, (4.1)], [22, Exercise 5.23]. The formula is given in terms of $\sigma(z)$ in [53, p.451, Example 3]. Rosengren [37, p.425] traced the formula back to Tannery and Molk [49, p.34]. Another $n$-term generalization, which reduces for $n=3$ to (2.7) after application of (2.4), is associated with root system $D_{n-1}$, see [24, Lemma 4.14], [15, Lemma A.1], [37, (4.6)]. More complicated many-term identities of theta products are given by Kajihara \& Noumi [28, Theorem 1.3] and by Langer, Schlosser \& Warnaar [30, Theorem 1.1].

Of particular interest are the four-term cases of the above identities. Quite some four-term theta identities can be found on scattered places in literature, and they also arise as special cases of some identities in [22]. One may wonder if some of these identities are essentially the same (a question asked by a referee). In one important case the answer will be negative, see the Appendix.

Various proofs and applications of (2.7) were given in [9], [1], 8]. Schlosser [42, Remark 3.3 ] observed that (2.7) pops up in connection with a telescoping property of the special case $e=a^{2} /(b c d)$ of Bailey's ${ }_{6} \psi_{6}$ summation formula [22, (II.33)]. This was converted into a proof of (2.7) by Chu [10, Theorem 1.1]. In [40, p.948] formula (2.7) is used in the proof of a determinant evaluation associated to the affine root system of type $C$. In [12] a $3 \times 3$ determinant with theta function entries is evaluated, thus solving an open problem in 23. The determinant evaluation has (2.7) as a special case. In [44] the so-called quintuple product identity [22, Exercise 5.6] is derived from (2.7).

The second fundamental formula (1.2) and its variants can be written in a very compact form by using the notation (cf. (1.3))

$$
[a]:=\theta_{a}(w) \theta_{a}(x) \theta_{a}(y) \theta_{a}(z), \quad[a]^{\prime}:=\theta_{a}\left(w^{\prime}\right) \theta_{a}\left(x^{\prime}\right) \theta_{a}\left(y^{\prime}\right) \theta_{a}\left(z^{\prime}\right) .
$$

Then (the first one implies the others):

$$
\begin{array}{ll}
2[1]=[1]^{\prime}+[2]^{\prime}-[3]^{\prime}+[4]^{\prime}, & 2[2]=[1]^{\prime}+[2]^{\prime}+[3]^{\prime}-[4]^{\prime}, \\
2[3]=-[1]^{\prime}+[2]^{\prime}+[3]^{\prime}+[4]^{\prime}, & 2[4]=[1]^{\prime}-[2]^{\prime}+[3]^{\prime}+[4]^{\prime} .
\end{array}
$$

These are easily seen to be equivalent with [53, p.468, Example 1 and p.488, Example 7]:

$$
\begin{array}{lll}
{[1]+[2]=[1]^{\prime}+[2]^{\prime},} & {[1]+[3]=[2]^{\prime}+[4]^{\prime},} & {[1]+[4]=[1]^{\prime}+[4]^{\prime},} \\
{[1]-[2]=[4]^{\prime}-[3]^{\prime},} & {[1]-[3]=[1]^{\prime}-[3]^{\prime},} & {[1]-[4]=[2]^{\prime}-[3]^{\prime} .}
\end{array}
$$

Jacobi [27, p.507, formula (A)] first obtained (3.7) and then derived (3.6) from it.

For $x=y=z=w(1.2)$ implies [53, p.469, Example 4]

$$
\theta_{1}(z)^{4}+\theta_{3}(z)^{4}=\theta_{2}(z)^{4}+\theta_{4}(z)^{4} .
$$

The computation [46, Proposition 3] of the action of the Casimir operators in the representation of the Sklyanin algebra uses (3.6). 


\section{Proofs of the fundamental theta relations}

For completeness I recall here the short and elegant complex analysis proofs of the fundamental theta relations (2.7) and (2.8).

Proof of (2.7) (Baxter [3, p.460], see also [48, p.3]).

Consider the theta functions in (2.7) with nome $q^{2}$. For fixed $y, u, v$ we have to prove that

$$
F(x):=\frac{y v^{-1} \theta\left(x y, x / y, v u, v / u ; q^{2}\right)+y u^{-1} \theta\left(x v, x / v, u y, u / y ; q^{2}\right)}{\theta\left(x u, x / u, y v, y / v ; q^{2}\right)}
$$

is equal to -1 . For generic values of $y, u, v F(x)$ is a meromorphic function of $x$ on $\mathbb{C} \backslash\{0\}$. Then the numerator vanishes at all (generically simple) zeros $x=q^{2 k} u^{ \pm 1}(k \in \mathbb{Z})$ of the denominator. Indeed, for these values of $x$ the numerator equals

$$
\begin{aligned}
& y v^{-1} \theta\left(q^{2 k} u^{ \pm 1} y, q^{2 k} u^{ \pm 1} y^{-1}, v u, v u^{-1} ; q^{2}\right)+y u^{-1} \theta\left(q^{2 k} u^{ \pm 1} v, q^{2 k} u^{ \pm 1} v^{-1}, u y, u y^{-1} ; q^{2}\right) \\
& =q^{-2 k(k-1)} u^{\mp 2 k}\left(y v^{-1} \theta\left(u^{ \pm 1} y, u^{ \pm 1} y^{-1}, v u, v u^{-1} ; q^{2}\right)+y u^{-1} \theta\left(u^{ \pm 1} v, u^{ \pm 1} v^{-1}, u y, u y^{-1} ; q^{2}\right)\right)=0
\end{aligned}
$$

where we used (2.6) and (2.4). Thus $F$ is analytic in $x$ on $\mathbb{C} \backslash\{0\}$. Furthermore, $F\left(q^{2} x\right)=F(x)$ by (2.5). Hence $F$ is bounded. Thus the singularity of $F$ at 0 is removable and, by Liouville's theorem, $F$ is constant. Now check that $F(v)=-1$ by (2.4).

Whittaker \& Watson [53, p.451, Examples 1 and 5] obtain (2.7) from (3.3). They suggest a proof of (3.3) by comparing zeros and poles of elliptic functions on both sides. Liu [31, (3.34)] proves (2.7) by using a kind of generalized addition formula for $\theta_{1}$.

Bailey [2, (5.2)] gives a more computational proof of (2.7). Among others he derives a threeterm identity [2, (4.6)] for very well-poised ${ }_{8} \phi_{7}$ series, which Gasper \& Rahman [22, Exercise 2.15] write in elegant symmetric form. By [22, Exercise 2.16] formula (2.7) then should follow from this three-term identity. Indeed, reduce it to a three-term identity of very well-poised ${ }_{6} \phi_{5}$ series which are summable by [22, (2.7.1)]. See also [22, Exercise 5.21].

Schlosser [41, after (4.2)] points out that (2.7) is also the special case $b=1$ of [22, (2.11.7)] (put $a=q x v, c=x y, d=q x / y, e=q v u, f=q v / u$ and use (2.4) and (2.5)).

Proof of (2.8) (Whittaker \& Watson [53, p.468]).

Divide the right-hand side by the left-hand side and consider the resulting expression as a meromorphic function $F(w)$ of $w$ on $\mathbb{C} \backslash\{0\}$ (the other variables generically fixed) with possible simple poles at the zeros $\pm q^{k}(k \in \mathbb{Z})$ of $\theta\left(w^{2} ; q^{2}\right)$. Since $F(w)=F(-w)$ we can write $F(w)=$ $G\left(w^{2}\right)$, where $G$ is a meromorphic function on $\mathbb{C} \backslash\{0\}$ with possible simple poles at $q^{2 k}(k \in \mathbb{Z})$. We have $F(q w)=F(w)$ because, by (2.5),

$$
\begin{aligned}
\frac{\theta\left( \pm q^{-1} w^{\prime \prime} ; q^{2}\right)}{\theta\left(q^{2} w^{2} ; q^{2}\right)} & =\frac{ \pm q^{-1} x y z w \theta\left( \pm q w^{\prime \prime} ; q^{2}\right)}{\theta\left(w^{2} ; q^{2}\right)}, \\
\frac{ \pm x y z w \theta\left( \pm q^{2} x^{\prime \prime}, \pm q^{2} y^{\prime \prime}, \pm q^{2} z^{\prime \prime} ; q^{2}\right)}{\theta\left(q^{2} w^{2} ; q^{2}\right)} & =\frac{\theta\left( \pm x^{\prime \prime}, \pm y^{\prime \prime}, \pm z^{\prime \prime} ; q^{2}\right)}{\theta\left(w^{2} ; q^{2}\right)} .
\end{aligned}
$$


Hence $G\left(q^{2} u\right)=G(u)$. But then

$$
2 \pi i \operatorname{Res}_{u=q^{2 k}}\left(u^{-1} G(u)\right)=\int_{|u|=|q|^{2 k-1}} G(u) \frac{d u}{u}-\int_{|u|=|q|^{2 k+1}} G(u) \frac{d u}{u}=0 .
$$

Hence $G$ has no poles and similarly for $F$. Similarly as in the previous proof we conclude that $F$ is constant in $w$. By symmetry, $F$ is also constant in $x, y$ and $z$. Thus we have shown that

$$
\begin{aligned}
A \theta\left(w^{2}, x^{2}, y^{2}, z^{2} ; q^{2}\right)=\theta\left(w^{\prime \prime}, x^{\prime \prime}, y^{\prime \prime}, z^{\prime \prime} ; q^{2}\right)+\theta\left(-w^{\prime \prime},-x^{\prime \prime},-y^{\prime \prime},-z^{\prime \prime} ; q^{2}\right) \\
\quad+q^{-1} x y z w\left(\theta\left(q w^{\prime \prime}, q x^{\prime \prime}, q y^{\prime \prime}, q z^{\prime \prime} ; q^{2}\right)-\theta\left(-q w^{\prime \prime},-q x^{\prime \prime},-q y^{\prime \prime},-q z^{\prime \prime} ; q^{2}\right)\right),
\end{aligned}
$$

for some constant A. Put in (4.1) $w=x=q^{\frac{1}{2}}$ and $y=z=i q$. Then $w^{\prime \prime}=x^{\prime \prime}=-q^{2}$ and $y^{\prime \prime}=z^{\prime \prime}=q$ and

$$
A \theta\left(q, q,-q^{2},-q^{2} ; q^{2}\right)=\theta\left(q, q,-q^{2},-q^{2} ; q^{2}\right)+q^{2} \theta\left(q^{3}, q^{3},-q^{2},-q^{2} ; q^{2}\right) .
$$

Hence $A=2$ by (2.5).

The last part of this proof is a slight improvement compared to [53, p.468]. There it is first proved in [53, §21.2] (again by the same method) that

$$
\theta\left(q ; q^{2}\right)^{2} \theta\left(q z ; q^{2}\right)^{2}=\theta\left(-q ; q^{2}\right)^{2} \theta\left(-q z ; q^{2}\right)^{2}-q z \theta\left(-q^{2} ; q^{2}\right)^{2} \theta\left(-q^{2} z ; q^{2}\right)^{2},
$$

and hence, by putting $z=1$,

$$
\theta\left(q ; q^{2}\right)^{4}=\theta\left(-q ; q^{2}\right)^{4}-q \theta\left(-q^{2} ; q^{2}\right)^{4}
$$

Then the value of $A$ in the above proof is obtained by putting $w=x=y=z=q^{\frac{1}{2}}$ in (4.1) and comparing with (4.3).

Note that (4.2) and (4.3) are special cases of (2.8). Another special case of (2.8), only leaving two terms nonzero, was suggested by a referee:

$$
(w, x, y, z):=\left(i / z, q^{-1 / 2} / z,-i q^{1 / 2} / z, z\right) . \quad \text { Then } \quad\left(w^{\prime \prime}, x^{\prime \prime}, y^{\prime \prime}, z^{\prime \prime}\right)=\left(-1, q,-q^{-1}, z^{-4}\right) .
$$

Then (2.8) degenerates to

$$
2 \theta\left(-z^{-2}, q^{-1} z^{-2},-q z^{-2}, z^{2} ; q^{2}\right)=\theta\left(-1, q,-q^{-1}, z^{-4} ; q^{2}\right) .
$$

This can be independently proved by (2.1) together with repeated use of the standard identities

$$
\left(a, q a ; q^{2}\right)_{\infty}=(a ; q)_{\infty} \text { and }(a,-a ; q)_{\infty}=\left(a^{2} ; q^{2}\right)_{\infty}
$$

Of course, (4.4) can also be used to settle that $A=2$ in (4.1).

In Jacobi [27, pp. 505-507] and in Mumford [33, Ch. 1, §5] a different proof of (2.8) is given. It uses (2.3).

If we compare our proofs of (2.7) and (2.8) given above with each other then we see that in the proof of (2.7) it is not automatic that the possible simple poles have residue zero because there are two simple poles in each annulus to be considered. So we have to check there by computation that the numerator of $F(z)$ vanishes whenever the denominator vanishes. 


\section{Equivalence of the two fundamental theta relations}

Let us rewrite the first fundamental theta relation (2.7) as $F_{1}(x, y, u, v ; q)=0$, where

$$
F_{1}(x, y, u, v ; q):=\theta\left(x y, x / y, u v, u / v ; q^{2}\right)-\theta\left(x v, x / v, u y, u / y ; q^{2}\right)-u y^{-1} \theta\left(y v, y / v, x u, x / u ; q^{2}\right) .
$$

In the second fundamental theta relation (2.8) both sides are invariant under each of the transformations of variable $w \rightarrow-w, x \rightarrow-x, y \rightarrow-y, z \rightarrow-z$. Therefore we obtain an equivalent identity if we replace in (2.8) $\left(w^{2}, x^{2}, y^{2}, z^{2}\right)$ by $(x y, x / y, u v, u / v)$. Thus we can write (2.8) equivalently, in a form closer to (5.1), as $F_{2}(x, y, u, v ; q)=0$, where

$$
\begin{aligned}
& F_{2}(x, y, u, v ; q) \\
& \quad \begin{array}{l}
:=2 \theta\left(x y, x / y, u v, u / v ; q^{2}\right)-\theta\left(x v, x / v, u y, u / y ; q^{2}\right)-\theta\left(-x v,-x / v,-u y,-u / y ; q^{2}\right) \\
-q^{-1} x u\left(\theta\left(q x v, q x / v, q u y, q u / y ; q^{2}\right)-\theta\left(-q x v,-q x / v,-q u y,-q u / y ; q^{2}\right)\right) .
\end{array}
\end{aligned}
$$

Theorem 5.1. The formulas $F_{1}(x, y, u, v ; q)=0$ and $F_{2}(x, y, u, v ; q)=0$ are equivalent to each other because of the following identities:

$$
\begin{aligned}
& \begin{aligned}
F_{1}(x, y, u, v ; q)+F_{1}(-x, y,-u, v ; q)-x y F_{1}(q x, q y, u, v ; q)-x y F_{1}(-q x, q y,-u, v ; q) & =F_{2}(x, y, u, v ; q),
\end{aligned} \\
& F_{2}(x, y, u, v ; q)-u y^{-1} F_{2}(x, u, y, v ; q)=2 F_{1}(x, y, u, v ; q) .
\end{aligned}
$$

Proof For the proof of (5.3) substitute (5.1) in the left-hand side of (5.3). Then this left-hand side becomes

$$
\begin{aligned}
& \theta\left(x y, x / y, u v, u / v ; q^{2}\right)-x y \theta\left(q^{2} x y, x / y, u v, u / v ; q^{2}\right) \\
& +\theta\left(-x y,-x / y,-u v,-u / v ; q^{2}\right)-x y \theta\left(-q^{2} x y,-x / y,-u v,-u / v ; q^{2}\right) \\
& -\theta\left(x v, x / v, u y, u / y ; q^{2}\right)-\theta\left(-x v,-x / v,-u y,-u / y ; q^{2}\right) \\
& +x y\left(\theta\left(q x v, q x / v, q u y, q^{-1} u / y ; q^{2}\right)+\theta\left(-q x v,-q x / v,-q u y,-q^{-1} u / y ; q^{2}\right)\right),
\end{aligned}
$$

which equals the right-hand side of (5.3) because of (2.5) and (5.2).

For the proof of (5.4) substitute (5.2) in the left-hand side of (5.4). Then

$$
\begin{aligned}
& 2 \theta\left(x y, x / y, u v, u / v ; q^{2}\right)-2 u y^{-1} \theta\left(x u, x / u, y v, y / v ; q^{2}\right) \\
& -\theta\left(x v, x / v, u y, u / y ; q^{2}\right)+u y^{-1} \theta\left(x v, x / v, u y, y / u ; q^{2}\right) \\
& -\theta\left(-x v,-x / v,-u y,-u / y ; q^{2}\right)+u y^{-1} \theta\left(-x v,-x / v,-u y,-y / u ; q^{2}\right) \\
& -q^{-1} x u\left(\theta\left(q x v, q x / v, q u y, q u / y ; q^{2}\right)-\theta\left(q x v, q x / v, q u y, q y / u ; q^{2}\right)\right) \\
& +q^{-1} x u\left(\theta\left(-q x v,-q x / v,-q u y,-q u / y ; q^{2}\right)-\theta\left(-q x v,-q x / v,-q u y,-q y / u ; q^{2}\right)\right),
\end{aligned}
$$

which equals the right-hand side of (5.4) because of (2.4), (2.5) and (5.1).

One half of the equivalence proof, i.e., essentially (5.4), has been given before by R. J. Chapman [9]. 
Remark 5.2. It would be interesting to see if the above equivalence extends to theta functions in several variables (cf. [50], [52] and [34, §21.6(i)]). Similarly the question arises if for root systems $A_{n-1}$ and $D_{n-1}$ there is not only a first fundamental theta identity [37] but also a second fundamental identity, equivalent to the first one.

\section{A Appendix: four-term theta identities}

Consider the case $n=4$ of $(3.5)$ :

$$
\sum_{k=1}^{4} \frac{\prod_{j=1}^{4} \theta\left(a_{k} / b_{j}\right)}{\prod_{j \neq k} \theta\left(a_{k} / a_{j}\right)}=0 \quad\left(a_{1} \ldots a_{4}=b_{1} \ldots b_{4}\right) .
$$

This can be seen as a four-term identity essentially depending on six free variables with each term having seven theta factors in the numerator and none in the denominator. Slater [47] mentions that (A.1) can be rewritten as her formula (3), which is reproduced in 22, Exercise 5.22]. As also mentioned in [47], replacement of $b, g, h$ by $a b, a g, a h$, respectively, let $a$ disappear from this formula. We are left with the four-term theta identity

$$
\begin{aligned}
b \theta(c b, d b, e b, f b, g, h, g / h ; q) & -b \theta(c h, d h, e h, f h, b, g, g / b ; q) \\
& =g \theta(c g, d g, e g, f g, b, h, b / h ; q)-h \theta(c, d, e, f, b / h, g / h, g / b ; q)
\end{aligned}
$$

under the side condition

$$
\text { bcdefgh }=q^{2} .
$$

A referee raised the question, to be answered negatively, whether (A.1) is essentially the same identity as the case $n=1$ of the elliptic ${ }_{12} V_{11}$ Frenkel-Turaev [21] identity, see [22, (11.2.23)], or equivalently (as pointed out by the same referee) as the case $b=1$ of Bailey's four-term identity [22, (2.12.9)] or [22, (III.39)] with side conditions there on p.58 or p.366, respectively.

First consider the case $n=1$ of [22, (11.2.23)]. While taking into account the side condition $\lambda=q a^{2} /(b c d)$ as well as formulas (11.2.19) and (11.2.5) in [22], and after substitution of $\theta(q ; p)=$ $-q \theta\left(q^{-1} ; p\right)$, the mentioned identity becomes the following explicit four-term theta identity:

$$
\begin{gathered}
1-\frac{\theta\left(b, c, d, e, f,(q a)^{3} /(b c d e f) ; p\right)}{\theta\left(q a / b, q a / c, q a / d, q a / e, q a / f, b c d e f /(q a)^{2} ; p\right)}=\frac{\theta\left(q a, q a /(e f),(q a)^{2} /(b c d e),(q a)^{2} /(b c d f) ; p\right)}{\theta\left(q a / e, q a / f,(q a)^{2} /(b c d e f),(q a)^{2} /(b c d) ; p\right)} \\
\times\left(1-\frac{\theta\left(q a /(c d), q a /(b d), q a /(b c), e, f,(q a)^{3} /(b c d e f) ; p\right)}{\theta\left(q a / b, q a / c, q a / d,(q a)^{2} /(b c d e),(q a)^{2} /(b c d f), e f /(q a) ; p\right)}\right) .
\end{gathered}
$$

Replace $a$ by $q^{-1} a$. This will eliminate $q$. Next replace $p$ by $q$. We get:

$$
\begin{aligned}
& 1-\frac{\theta\left(b, c, d, e, f, a^{3} /(b c d e f) ; q\right)}{\theta\left(a / b, a / c, a / d, a / e, a / f, b c d e f / a^{2} ; q\right)}=\frac{\theta\left(a, a /(e f), a^{2} /(b c d e), a^{2} /(b c d f) ; q\right)}{\theta\left(a / e, a / f, a^{2} /(b c d e f), a^{2} /(b c d) ; q\right)} \\
& \times\left(1-\frac{\theta\left(a /(c d), a /(b d), a /(b c), e, f, a^{3} /(b c d e f) ; q\right)}{\theta\left(a / b, a / c, a / d, a^{2} /(b c d e), a^{2} /(b c d f), e f / a ; q\right)}\right) .
\end{aligned}
$$


Now multiply both sides by $\theta\left(a / b, a / c, a / d, a / e, a / f, b c d e f / a^{2}, a^{2} /(b c d) ; q\right)$ and simplify by using (2.4). We obtain:

$$
\begin{array}{r}
\theta\left(a / b, a / c, a / d, a / e, a / f, b c d e f / a^{2}, a^{2} /(b c d) ; q\right)-\theta\left(b, c, d, e, f, a^{3} /(b c d e f), a^{2} /(b c d) ; q\right) \\
=-a^{-2} b c d e f \theta\left(a, a /(e f), a^{2} /(b c d e), a^{2} /(b c d f), a / b, a / c, a / d ; q\right) \\
-a^{-1} b c d \theta\left(a, a /(c d), a /(b d), a /(b c), e, f, a^{3} /(b c d e f) ; q\right) .
\end{array}
$$

Next consider [22, (III.39)] with side conditions given there on p.366. Its case $b=1$ takes by use of (2.1) and (2.4) the form

$$
\begin{aligned}
1-a^{-1} \frac{\theta(c, d, e, f, g, h ; q)}{\theta(c / a, d / a, e / a, f / a, g / a, h / a ; q)}=-\lambda \frac{\theta\left(a^{-1}, f / \lambda, g / \lambda, h / \lambda ; q\right)}{\theta(\lambda, f / a, g / a, h / a ; q)} & +\frac{\theta\left(a^{-1}, f, g, h, \lambda c / a, \lambda d / a, \lambda e / a ; q\right)}{\theta(\lambda, c / a, d / a, e / a, f / a, g / a, h / a ; q)}
\end{aligned}
$$

or equivalently

$$
\begin{aligned}
\theta(c / a, d / a, e / a, f / a, g / a, h / a, \lambda ; q)-a^{-1} \theta(c, d, e, f, g, h, \lambda ; q) \\
\quad=-\lambda \theta\left(a^{-1}, f / \lambda, g / \lambda, h / \lambda, c / a, d / a, e / a ; q\right)+\theta\left(a^{-1}, f, g, h, \lambda c / a, \lambda d / a, \lambda e / a ; q\right) .
\end{aligned}
$$

The side conditions take for $b=1$ the form

$$
\lambda=\frac{q a^{2}}{c d e}, \quad h=\frac{a^{3} q^{2}}{c d e f g} .
$$

By substitution of these in the identity, the variables $\lambda$ and $h$ disappear from the identity. Now replace $e, b, g$ by $b, g, e$ (cyclic permutation) and use (2.4) and (2.6). We arrive at (A.4).

Both (A.4) and (A.2) (with side condition) have six free variables and seven theta factors in each term. However, in (A.2) each term has three theta factors with the property that the product of the arguments of two of them is equal to the argument of the third. On the other hand, in none of the terms of (A.4) there is such a simple relation between the arguments of the theta factors. Of course, they must satisfy a relation, but this is a more complicated polynomial relation involving the arguments of more than three of the theta factors. Therefore, (A.4) and (A.2) cannot be matched with each other.

Let us finally consider some other four-term theta identities. The case $n=4$ of the $D_{n-1}$ identity is a four-term identity depending on six free variables with each term having ten theta factors. A special case of both [28, Theorem 1.3] and [30, Theorem 1.1] is the four-term theta identity [30, (1.4)] with four free variables and each term having six theta factors. As pointed out to me by Michael Schlosser, a further specialization of this identity to two free variables yields identity (3.5) in Bouttier et al. [7] (take $w=z, x=v=1 / \alpha$ and $t=\alpha^{3}$ in [30, (1.4)]). The identity in [7] was obtained in a statistical mechanical context and it was proved in a way quite similar to Baxter's proof of (2.7) (see Section 44). As Hjalmar Rosengren pointed out to me, the $m=2$ cases of Pfaffian evaluations in [39, Remark 2.1] yield four-term identities of theta products as well. 


\section{References}

[1] G. E. Andrews, R. J. Baxter and P. J. Forrester, Eight-vertex SOS model and generalized Rogers-Ramanujan-type identities, J. Statist. Phys. 35 (1984), 193-266.

[2] W. N. Bailey, Series of hypergeometric type which are infinite in both directions, Quart. J. Math. Oxford Ser. 7 1936, 105-115.

[3] R. J. Baxter, Exactly solved models in statistical mechanics, Academic Press, 1982.

[4] B. C. Berndt, Ramanujan's notebooks, Part 3, Springer-Verlag, 1991.

[5] M. Bonk, The characterization of theta functions by functional equations. Abh. Math. Sem. Univ. Hamburg 65 (1995), 29-55.

[6] U. Bottazzini and J. Gray, Hidden harmony-geometric fantasies. The rise of complex function theory, Springer-Verlag, 2013.

[7] J. Bouttier, P. Di Francesco and E. Guitter, Random trees between two walls: exact partition function, J. Phys. A 36 (2003), 12349-12366.

[8] S. H. Chan, A new proof of Winquist's identity, in Ramanujan rediscovered, Ramanujan Math. Soc. Lect. Notes Ser. 14, Ramanujan Math. Soc., Mysore, 2010, pp. 73-78.

[9] W. Chu, S. B. Ekhad and R. J. Chapman, A q-trigonometric identity: Solutions of Problem 10226, Amer. Math. Monthly 103 (1996), 175-177.

[10] W. Chu, Theta function identities and Ramanujan's congruences on the partition function, Quart. J. Math. 56 (2005), 491-506.

[11] W. Chu, Common source of numerous theta function identities, Glasgow Math. J. 49 (2007), $61-79$.

[12] S. Cooper and P. C. Toh, Determinant identities for theta functions, J. Math. Anal. Appl. 347 (2008), 1-7.

[13] E. Date, M. Jimbo, A. Kuniba, T. Miwa and M. Okado, Exactly solvable SOS models: local height probabilities and theta function identities, Nuclear Phys. B 290 (1987), 231-273.

[14] E. Date, M. Jimbo, A. Kuniba, T. Miwa and M. Okado, Exactly solvable SOS models. II. Proof of the star-triangle relation and combinatorial identities, in: Conformal field theory and solvable lattice models, Academic Press, 1988, pp. 17-122; reprinted in Yang-Baxter equation in integrable systems, World Scientific, 1989, pp. 498-614.

[15] J. E. van Diejen and V. P. Spiridonov, Elliptic Selberg integrals, Internat. Math. Res. Notices (2001), no. 20, 1083-1110. 
[16] B. A. Dubrovin, Theta-functions and nonlinear equations, Russian Math. Surveys 36 (1981), $11-92$.

[17] L. Ehrenpreis and R. C. Gunning (eds.), Theta functions-Bowdoin 1987, Proceedings of Symposia in Pure Mathematics 49, Parts 1 and 2, Amer. Math. Soc., 1989.

[18] A. Erdélyi et al., Higher transcendental functions, Vol. 2, McGraw-Hill, 1953.

[19] G. Felder, Elliptic quantum groups, in: XIth International Congress of Mathematical Physics (Paris, 1994), International Press, 1995, pp. 211-218; arXiv:hep-th/9412207v1.

[20] G. Felder and A. Varchenko, On representations of the elliptic quantum group $E_{\tau, \eta}\left(s l_{2}\right)$, Comm. Math. Phys. 181 (1996), 741-761.

[21] I. B. Frenkel and V. G. Turaev, Elliptic solutions of the Yang-Baxter equation and modular hypergeometric functions, in The Arnold-Gelfand mathematical seminars, Birkhäuser, 1997, 171-204.

[22] G. Gasper and M. Rahman, Basic hypergeometric series, second ed., Cambridge University Press, 2004.

[23] R. Wm. Gosper and R. Schroeppel, Somos sequence near-addition formulas and modular theta functions, arXiv:math/0703470v1, 2007.

[24] R. A. Gustafson, Multilateral summation theorems for ordinary and basic hypergeometric series in $U(n)$, SIAM J. Math. Anal. 18 (1987), 1576-1596.

[25] T. Hawkins, The mathematics of Frobenius in context, Springer-Verlag, 2013.

[26] A. Hurwitz, Über die Weierstraßsche $\sigma$-Funktion, in: Mathematische Abhandlungen Hermann Amandus Schwarz zu seinem fünfzigjährigen Doktorjubiläum am 6. August 1914 gewidmet von Freunden und Schülern, Springer-Verlag, 1914, pp. 133-141.

[27] C. G. J. Jacobi, Theorie der elliptischen Functionen aus den Eigenschaften der Thetareihen abgeleitet, in Gesammelte Werke, Erster Band (herausgegeben von C. W. Borchardt), G. Reimer, Berlin, 1881, pp. 497-538.

[28] Y. Kajihara and M. Noumi, Multiple elliptic hypergeometric series. An approach from the Cauchy determinant, Indag. Math. (N.S.) 14 (2003), 395-421.

[29] E. Koelink, Y. van Norden and H. Rosengren, Elliptic U(2) quantum group and elliptic hypergeometric series, Comm. Math. Phys. 245 (2004), 519-537.

[30] R. Langer, M. J. Schlosser and S. O. Warnaar, Theta functions, elliptic hypergeometric series, and Kawanaka's Macdonald polynomial conjecture, SIGMA 5 (2009), 055, 20 pp.; arXiv:0905.4033v1. 
[31] Z.-G. Liu, An addition formula for the Jacobian theta function and its applications, Adv. Math. 212 (2007), 389-406.

[32] D. Mumford, On the equations defining abelian varieties. I, Invent. Math. 1 (1966), 287-354.

[33] D. Mumford, Tata lectures on theta I, Progress in Math. 28, Birkhäuser, 1983; reprinted 2008.

[34] F. W. J. Olver et al., NIST Handbook of Mathematical Functions, Cambridge University Press, 2010; DLMF, Digital Library of Mathematical Functions, http://dlmf.nist.gov.

[35] B. Riemann, Theorie der Abel'schen Functionen, J. Reine Angew. Math. 54 (1857), 115-156.

[36] B. Riemann, Gesammelte mathematische Werke und wissenschaftlicher Nachlass (herausgegeben von H. Weber), Teubner, Leipzig, 1876.

[37] H. Rosengren, Elliptic hypergeometric series on root systems, Adv. Math. 181 (2004), 417447.

[38] H. Rosengren, An elementary approach to $6 j$-symbols (classical, quantum, rational, trigonometric, and elliptic), Ramanujan J. 13 (2007), 131-166.

[39] H. Rosengren, Sums of triangular numbers from the Frobenius determinant, Adv. Math. 208 (2007), 935-961.

[40] H. Rosengren and M. Schlosser, Elliptic determinant evaluations and the Macdonald identities for affine root systems, Compos. Math. 142 (2006), 937-961.

[41] M. Schlosser, Elementary derivations of identities for bilateral basic hypergeometric series, Selecta Math. (N.S.) 9 (2003), 119-159.

[42] M. Schlosser, Inversion of bilateral basic hypergeometric series, Electron. J. Combin. 10 (2003), Research Paper 10, 27 pp.

[43] H. A. Schwarz, Formeln und Lehrsätze zum Gebrauche der elliptischen Functionen, Erste Abteilung (nach Vorlesungen und Aufzeichnungen des Herrn Professor K. Weierstrass), Göttingen, 1881-1883; Zweite Ausgabe, Julius Springer, Berlin, 1893.

[44] D. B. Sears, Two identities of Bailey, J. London Math. Soc. 27 (1952), 510-511.

[45] E. K. Sklyanin, Some algebraic structures connected with the Yang-Baxter equation, Functional Anal. Appl. 16 (1982), 263-270.

[46] E. K. Sklyanin, Some algebraic structures connected with the Yang-Baxter equation. Representations of a quantum algebra, Functional Anal. Appl. 17 (1983), 273-284.

[47] L. J. Slater, A note on equivalent product theorems, Math. Gazette 38 (1954), 127-128. 
[48] V. P. Spiridonov, Elliptic hypergeometric functions, arXiv:0704.3099v1 [math.CA], 2007; a complement to the book by G. E. Andrews, R. Askey, and R. Roy, Special Functions, Cambridge Univ. Press, 1999, written for its Russian edition.

[49] J. Tannery and J. Molk, Éléments de la théorie des fonctions elliptiques, Tome III: Calcul intégral, Gauthier-Villars, Paris, 1898.

[50] K. Weierstrass, Zur Theorie der Jacobischen Funktionen von mehreren Veränderlichen, Sitzungsber. Königl. Preuss. Akad. Wiss. (1882), 505-508; Werke, Band 3, pp. 155-159.

[51] K. Weierstrass, Vorlesungen über die Theorie der Abelschen Transcendenten (bearbeitet von G. Hettner und J. Knoblauch), Mayer \& Müller, Berlin, 1902.

[52] K. Weierstrass, Verallgemeinerung einer Jacobischen Thetaformel, in: Mathematische Werke, Band 3, Mayer \& Müller, Berlin, 1903, pp. 123-137.

[53] E. T. Whittaker and G. N. Watson, A course of modern analysis, fourth ed., Cambridge University Press, 1927.

T. H. Koornwinder, Korteweg-de Vries Institute, University of Amsterdam, P.O. Box 94248, 1090 GE Amsterdam, The Netherlands;

email: T.H.Koornwinder@uva.nl 